\title{
Entscheidung für den Lebensabend: Vorbereitung der Entscheidungen zu Patientenverfügung und Vorsorgevollmacht
}

Thomas Weiß

(C) Springer-Verlag Wien 2012

In einer Patientenverfügung wird inhaltlich festgelegt, was geschehen soll oder auch nicht. In einer Vorsorgevollmacht wird eine andere Person berechtigt, für den Vollmachtgeber in Vertretung rechtswirksam zu handeln und dessen Willen dann durchzusetzen, wenn er selbst dazu nicht (mehr) in der Lage ist.

Obwohl zur Vorsorge eine Kombination dieser Instrumente empfohlen wird, werden sie leider noch nicht ausreichend genutzt.

Wir leben zwar auch ansonsten in einer von Selbstbestimmung und Ansprüchen auf Selbstbestimmung gekennzeichneten Kultur wie nie zuvor, aber die Angst vor dem Tode, mehr noch vor dem Sterben, verursacht einen Bruch in unserem Selbstbeststimmungsverhalten. Wir sorgen für Situationen vor, die erst nach unserem Tode Bedeutung gewinnen (etwa in Testamenten). Nur in Bezug auf die Art und Weise wie wir die letzten Stunden, Tage oder Wochen unseres Lebens verleben möchten verzichten viele von uns auf Selbstbestimmung. Auch Angehörige tun sich schwer - und wie sollen Mediziner und Pflegekräfte das Heil und Wohl bestimmen oder sich an unserem Willen orientieren, wenn man den Willen oder Wunsch nicht oder nicht eindeutig erkennen kann und ein Pflegebedürftiger sich in dieser Situation auch nicht mehr verständlich machen kann? Ist es da nicht am sichersten, medizinisch alles zu tun, was das biologische Leben nicht beendet?

Deshalb sollte man auch insoweit vorsorgen und in einer Patientenverfügung deutlich machen, was man insbesondere am Lebensende will, auch um die Last der Entscheidung den Angehörigen und Ärzten weitgehend zu nehmen.

Patientenverfügungen können allerdings niemals so eindeutig sein, dass nicht Spielräume für Auslegungsmöglichkeiten bleiben. Hier hilft ein Bevollmächtigter, der ggf. Zweifel ausräumen kann, auch darüber, ob der Betroffene über Bedeutung und Tragweite seiner Entscheidung ausreichend aufgeklärt war. Und nach einem gemeinsamen Gespräch zwischen behandelndem Arzt und Bevollmächtigten wird dann ,im Hinblick auf den Gesamtzustand und die Prognose des Patienten ... unter Berücksichtigung des Patientenwillens als Grundlage“ ( $\$ 1901$ b BGB) gehandelt.

T. Weiß $(\square)$

Rechtsanwaltskanzlei Weiß und Kreitz, Kiel 